\title{
Static Compensator for Nonsquare Systems - Application Example
}

\author{
Daniel Honc, František Dušek and Jan Merta \\ University of Pardubice, Pardubice 532 10, Czech Republic \\ daniel.honc@upce.cz
}

\begin{abstract}
The paper deals with the decentralized control of multivariable systems with the number of manipulated variables greater than the number of controlled variables. Proposed static compensator ensures automatic creation of input/output pairs for the simple control loops. The compensator provides steadystate autonomy and unit gain of the controlled system. Steady-state gain matrix and vector of the offsets are enough information for the compensator design. Laboratory example is presented to demonstrate innovative compensator design and its application.
\end{abstract}

Keywords: multivariable system, decentralized control, static compensator.

\section{Introduction}

One set-point change and subsequent manipulated variable change acts as a disturbance to the other control loops in case of Multi-Input Multi-Output (MIMO) system control. Two approaches for controlling MIMO systems are possible - see Fig. 1.
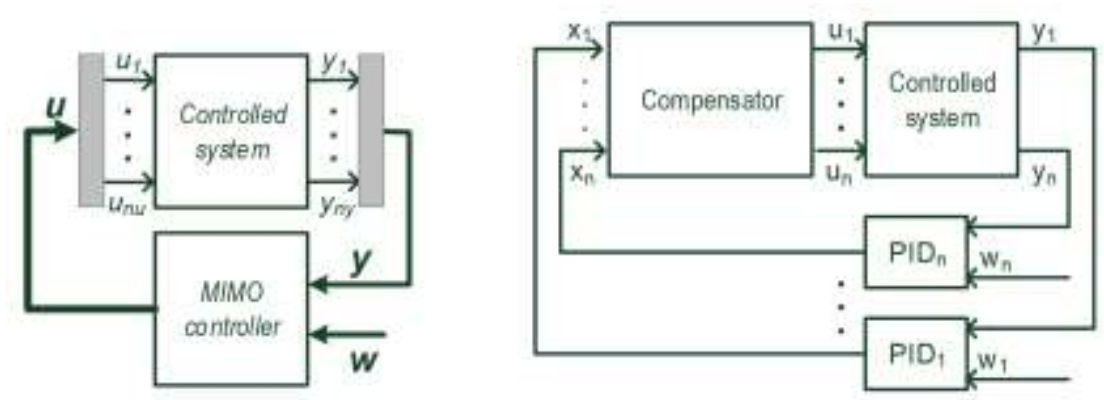

Fig. 1. Multivariable controller and decentralized control.

Multivariable controller calculates the values of all manipulated variables simultaneously from all controlled variables and set-points. Special block of the compensator can be placed in front of the controlled system to ensure the autonomy of the controlled system in case of the decentralized control. Dynamical autonomy of square systems means that changing in input $\mathrm{u}_{\mathrm{i}}$ will only cause a change to the corresponding output 
$y_{\mathrm{i}}$. It is necessary to know linear dynamical model of the controlled system. Dynamic compensator ensuring autonomy in transient states can be complex and not physically feasible [1], [2]. Because of this only limited autonomy compensators are designed. For the practical applications of decentralized control even without the compensator the input/output pairing and control loop tuning can be challenging task.

The paper deals with the specific type of autonomous control of the systems with more inputs than outputs. Steady state autonomy is realized with innovative static compensator - see Fig. 2.

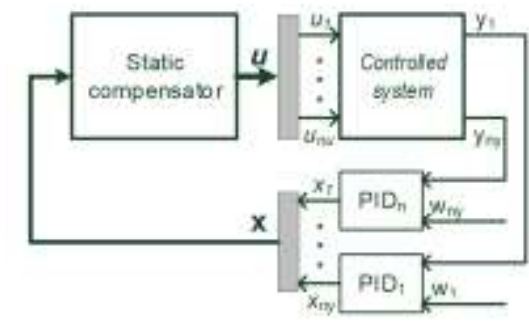

Fig. 2. Decentralized control with static compensator.

The introduction of new manipulated variables $\mathrm{x}_{\mathrm{i}}$ assigned to the corresponding controlled variables $y_{i}$ solves the problem of creating the control loops. The new control variables (vector $\mathbf{x}$ ) are recalculated by the static compensator to the system inputs (vector $\mathbf{u}$ ). The gain matrix between the new control variables and the system outputs (vector $\mathbf{y}$ ) is unit matrix - in steady state $\mathbf{y}=\mathbf{x}$. Some disadvantage can be that the use of a static compensator may make worse the frequency properties of the closed loop control circuit [5], [6]. The proposed static compensator design is a general procedure for the application of the multivariable Split Range method [7]. Controlled process gain matrix estimation and compensator design was published in [3].

In the paper gain matrix estimation in Excel and laboratory application to a process with four inputs and three outputs and compact control system AMiNi4DS is presented.

\section{Gain matrix estimation}

Experimental data - steady-state output values for different input combinations are needed to estimate the gain matrix of the controlled system. The number of the samples must be greater than the number of the inputs. The method is described in detail in [3].

Steady-state model of the controlled process with inputs $\mathbf{u}\left(\mathbf{u}: n_{\mathbf{u}} \times 1\right)$ and outputs $\mathbf{y}$ $\left(\mathbf{y}: n_{\mathrm{y}} \times 1\right)$ has a form

$$
\mathbf{y}=\mathbf{Z} \cdot \mathbf{u}+\mathbf{z}_{0}
$$

where $\mathbf{Z}$ is the gain matrix $\left(\mathbf{Z}: n_{\mathrm{y}} \times n_{\mathrm{u}}\right)$ and

$\mathbf{z}_{0}$ is an offset vector $\left(\mathbf{z}_{0}: n_{\mathrm{y}} \times 1\right)$. 
The gain matrix $\mathbf{Z}$ and the vector of the offsets $\mathbf{z}_{0}$ is estimated by least square matrix method from $N$ samples of the steady state input/outputs pairs of the controlled system. We arrange the data into a matrix of output values $\mathbf{Y}\left(\mathbf{Y}: N \times n_{\mathrm{y}}\right)$ and extended matrix of input values $\mathbf{U}_{\mathrm{r}}\left(\mathbf{U}_{\mathrm{r}}: N \times n_{\mathrm{u}}+1\right)$ as follows

$$
\mathbf{Y}=\left[\begin{array}{c}
\mathbf{y}^{T}(1) \\
\vdots \\
\mathbf{y}^{T}(N)
\end{array}\right]=\left[\begin{array}{ccc}
y_{1}(1) & \cdots & y_{n y}(1) \\
\vdots & \ddots & \vdots \\
y_{1}(N) & \cdots & y_{n y}(N)
\end{array}\right], \quad \mathbf{U}_{r}=\left[\begin{array}{cc}
\mathbf{u}^{T}(1) & 1 \\
\vdots & \vdots \\
\mathbf{u}^{T}(N) & 1
\end{array}\right]=\left[\begin{array}{cccc}
u_{1}(1) & \cdots & u_{n u}(1) & 1 \\
\vdots & \ddots & \vdots & \vdots \\
u_{1}(N) & \cdots & u_{n u}(N) & 1
\end{array}\right]
$$

Solution - the extended $\mathbf{Z}_{\mathrm{r}}$ gain matrix is obtained as

$$
\mathbf{Z}_{r}=\mathbf{Y}^{T} \cdot \mathbf{U}_{r} \cdot\left(\mathbf{U}_{r}^{T} \cdot \mathbf{U}_{r}\right)^{-1}
$$

The gain matrix $\mathbf{Z}$ is the first $n_{\mathrm{u}}$ columns and the vector of the offsets $\mathbf{z}_{0}$ is the last column of the extended matrix $\mathbf{Z}_{\mathrm{r}}$.

\section{Static compensator design}

Compensator together with the original system create new system with the same number of new inputs $\mathbf{x}\left(\mathbf{x}: n_{\mathrm{y}} \times n_{\mathrm{y}}\right)$ as the outputs $\mathbf{y}$. The gain matrix of the new system is unit matrix I $\left(\mathbf{I}: n_{\mathrm{y}} \times n_{\mathrm{y}}\right)$

$$
\mathbf{y}_{\infty}=\mathbf{Z} . \mathbf{u}+\mathbf{z}_{0}, \quad \mathbf{u}=\mathbf{K} . \mathbf{x}, \quad \mathbf{y}_{\infty}=\mathbf{x}
$$

In case of the systems, where the number of inputs is greater than the number of outputs, there are infinity combinations of inputs leading to the desired outputs. It is possible to introduce an additional requirement (preferred, desired vector of inputs $\mathbf{u}_{\mathrm{w}}$ ). Compensator calculation is done by minimizing the deviation of the input vector $\mathbf{u}$ from the preferred input vector $\mathbf{u}_{\mathrm{w}}$, with the constraint respecting the relationship between inputs and outputs $\mathbf{y}=\mathbf{Z} . \mathbf{u}+\mathbf{z}_{0}$ and the requirement of the unit gain $\mathbf{y}_{\infty}=\mathbf{I} . \mathbf{x}$

$$
\begin{aligned}
\min _{\mathbf{u}} J(\mathbf{u}), \quad J(\mathbf{u}) & =\left(\mathbf{u}-\mathbf{u}_{w}\right)^{T} \cdot \mathbf{M} \cdot\left(\mathbf{u}-\mathbf{u}_{w}\right) \\
\text { subject to }: \mathbf{y}_{\infty} & =\mathbf{Z} \cdot \mathbf{u}+\mathbf{z}_{0}=\mathbf{I} \cdot \mathbf{x}
\end{aligned}
$$

where the optional matrix $\mathbf{M}\left(\mathbf{M}: n_{\mathrm{u}} \times n_{\mathrm{u}}\right)$ includes possible weighting requirements for the individual inputs or their combinations (it can be chosen as an unit matrix). The matrix $\mathbf{M}$ and the vector of the preferred inputs $\mathbf{u}_{\mathrm{w}}$ allows to include additional requirements to the control - in addition to the set-points following.

The minimization of the cost function (5) is described in detail in [3] and the solution is

$$
\left[\begin{array}{l}
\mathbf{u} \\
\boldsymbol{\lambda}
\end{array}\right]=\left[\begin{array}{cc}
\mathbf{M}^{T} & \frac{1}{2} \mathbf{Z}^{T} \\
\mathbf{Z} & 0
\end{array}\right]^{-1} \cdot\left[\begin{array}{c}
\mathbf{M}^{T} \cdot \mathbf{u}_{w} \\
\mathbf{y}_{\infty}-\mathbf{z}_{0}
\end{array}\right]
$$


Let us use the equality $\mathbf{y}_{\infty}=\mathbf{x}$, introduce the notation $\mathbf{S}\left(\mathbf{S}: n_{\mathrm{u}}+n_{\mathrm{y}} \times n_{\mathrm{u}}+n_{\mathrm{y}}\right)$ for the inverse matrix and denote two submatrices $\mathbf{R}\left(\mathbf{R}: n_{\mathrm{u}} \times n_{\mathrm{u}}\right)$ and $\mathbf{K}\left(\mathbf{K}: n_{\mathrm{u}} \times n_{\mathrm{y}}\right)$ as indicated in the equation

$$
\left[\begin{array}{l}
\mathbf{u} \\
\boldsymbol{\lambda}
\end{array}\right]=\mathbf{S} \cdot\left[\begin{array}{c}
\mathbf{M}^{T} . \mathbf{u}_{w} \\
\mathbf{x}-\mathbf{z}_{0}
\end{array}\right], \quad \mathbf{S}=\left[\begin{array}{cc}
\mathbf{R} & \mathbf{K} \\
\mathbf{X}_{1} & \mathbf{X}_{2}
\end{array}\right]
$$

Since we are interested in the solution with respect to the vector $\mathbf{u}$, we will only use the submatrices $\mathbf{R}$ and $\mathbf{K}$. The static compensator can be written in the matrix form as

$$
\mathbf{u}=\mathbf{K} \cdot\left(\mathbf{x}-\mathbf{z}_{0}\right)+\mathbf{R} \cdot \mathbf{M}^{T} \cdot \mathbf{u}_{w}
$$

and its block scheme is in Fig. 3.

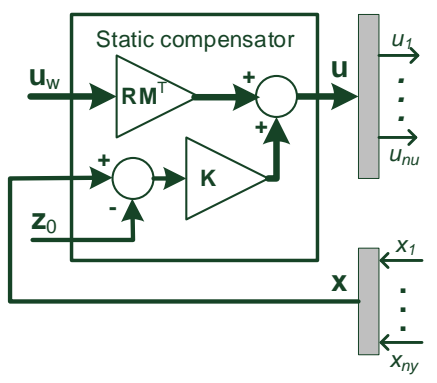

Fig. 3. Static compensator.

\section{Laboratory application}

The calculation of the static compensator in Microsoft Excel and the program implementation with standard PID controllers in the compact control system AMiNi4DS is presented on example of a system with four inputs and three outputs. 15 steady-state input/output pairs were measured for the gain matrix estimation. A hardware-based simulator of the dynamic systems was used as a controlled system for the application.

\subsection{Gain matrix estimation in Excel}

Example of the static compensator calculation in Excel is shows in Fig. 4. The progress of the solution is indicated by arrows with the numbers of each calculation step. The measured values in the Measured data area are supplemented with a column of ones. Auxiliary matrices are calculated in the steps 1 to 3 and calculation of the extended gain matrix $\mathbf{Z}_{\mathrm{r}}$ according to the equation (2) is completed the in the step 4 . This matrix contains the process gains $\mathbf{Z}$ and the offsets $\mathbf{z}_{0}$. The offsets $\mathbf{z}_{0}$ can be updated at any time (process outputs for zero inputs) without having to recalculate the compensator matrices which depends only on the gain matrix $\mathbf{Z}$. 


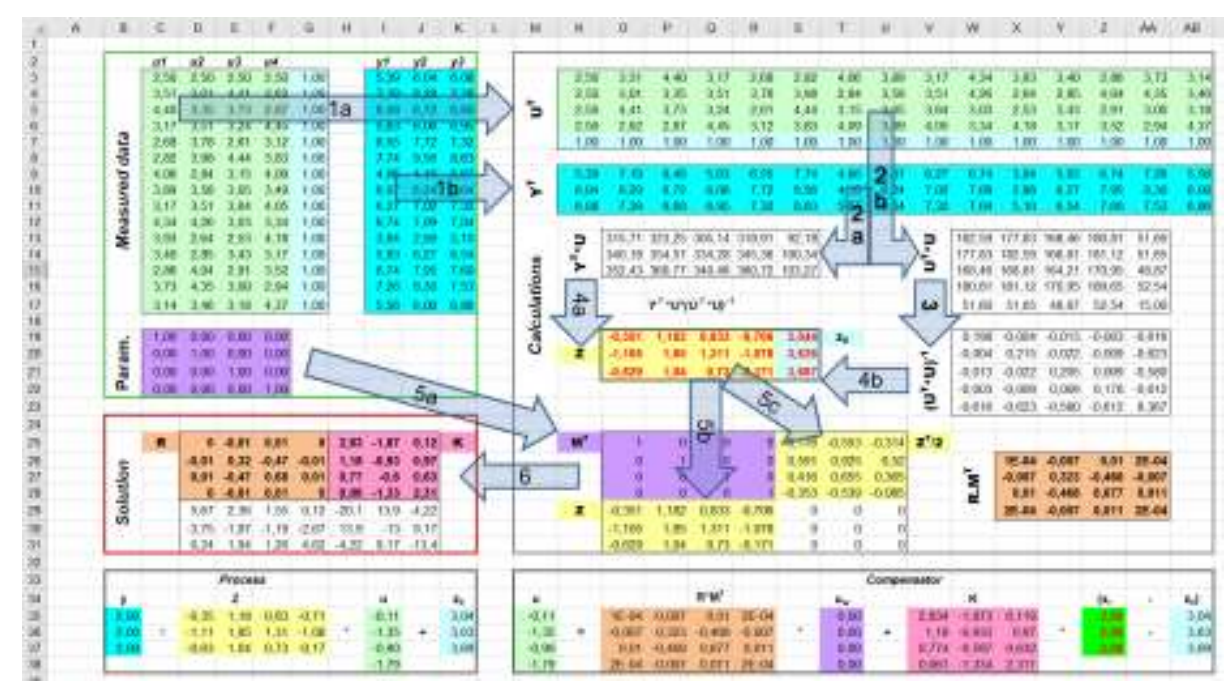

Fig. 4. Calculations in Excel.

Auxiliary matrix $\mathbf{S}$ for the calculation of the compensator in the step 5 according to the equation (6) is based on the gain matrix $\mathbf{Z}$ of the process and the chosen weighting matrix $\mathbf{M}$ (area Param.). In the last step 6 the compensator matrices $\mathbf{K}, \mathbf{R}$ and the $\mathbf{R} \mathbf{M}^{\mathrm{T}}$ matrix for the implementation of the compensator is calculated. The static compensator represents a matrix equation (7) for the recalculation of the vector $\mathbf{x}$ (3 outputs of the PID controllers) to vector $\mathbf{u}$ ( 4 inputs of the controlled system) respecting the system offset $\mathbf{z}_{0}$ and the vector $\mathbf{u}_{\mathrm{w}}$ of the preferred input vector.

\subsection{Application in the control system AMiNi4DS}

An application of the proposed compensator is presented in the compact control system AMiNi4DS. This control system supports matrix multiplication and provides also digital PID controllers so the control including a static compensator is easy to implement.

Static compensator and three PID controllers were realized programmatically using the design environment supplied with the control system according to the scheme in Fig. 5. The $\mathbf{R}_{\mathrm{m}}$ and $\mathbf{K}$ matrices used by the compensator were manually filled with the values calculated in Excel. The offset vector $\mathbf{z}_{0}$ and the preferred input vector $\mathbf{u}_{\mathrm{w}}$ can be changed from the control panel.

Controllers and the static compensators are calculated in one process. Part of the program code in the form of structured text is shown in Fig. 6. Controller variables of the controllers (standard PID module) are directly connected to the analog inputs AI.02 and the set-points to inputs AI.4-6. Units for all inputs and outputs variables of the controllers Volts. The outputs of the PID controllers are merged into the vector $\mathbf{x}$. Vector $\mathbf{x}$ is recalculated according to the matrix equation of the static compensator to the vector $\mathbf{u}$ and split to the individual analog outputs AO.0-3. 


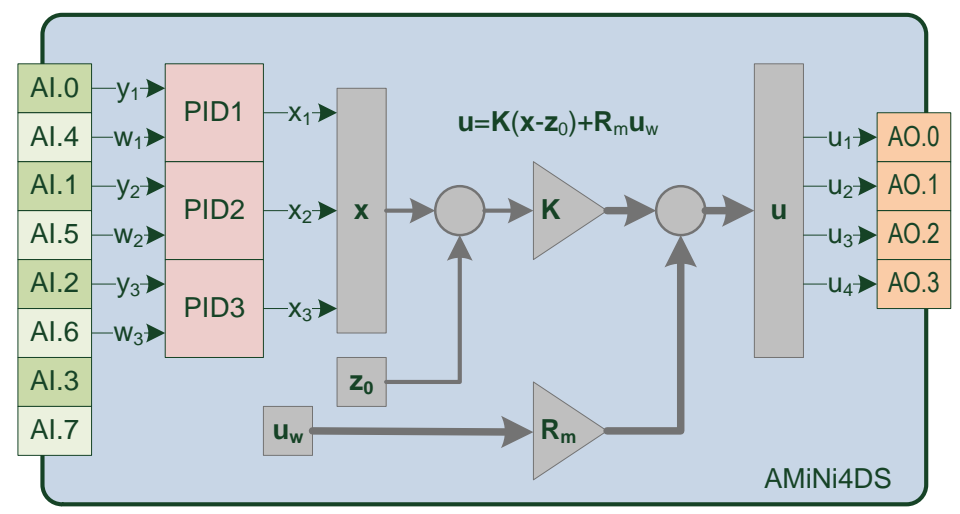

Fig. 5. Controllers and compensator in AMiNi4DS.

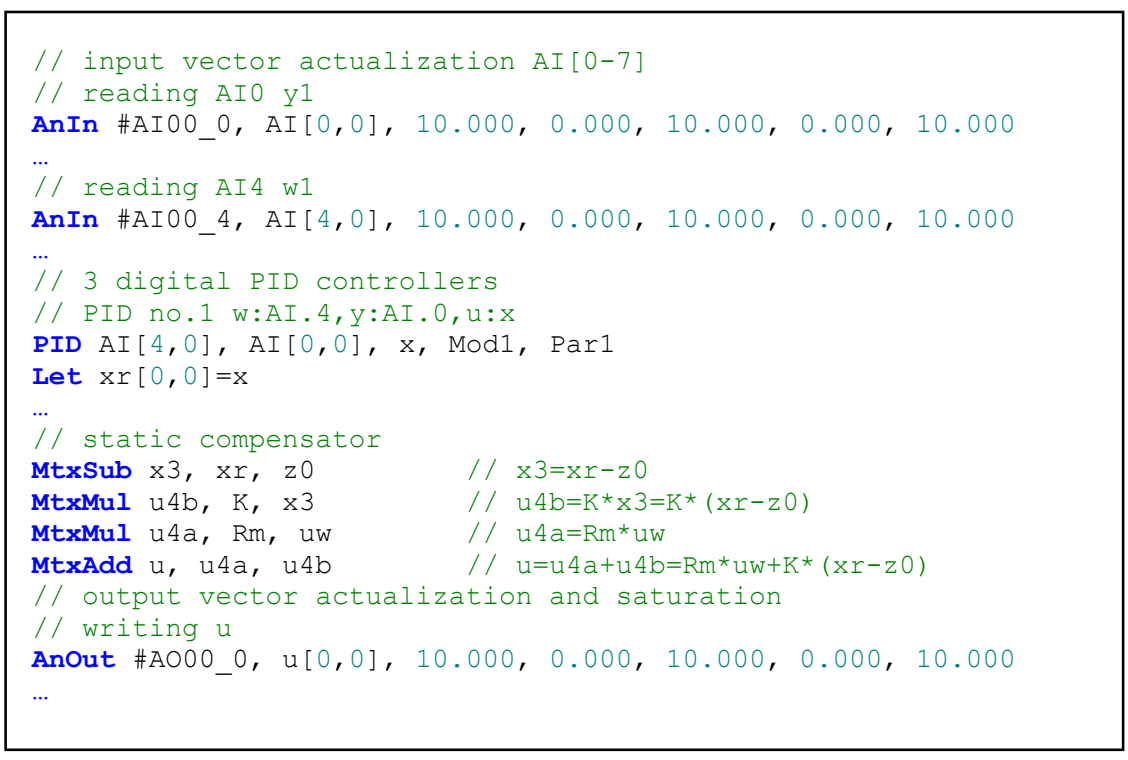

Fig. 6. Code in AMiNi4DS.

\section{Conclusions}

The paper describes design of an innovative simple and practically usable multivariable decentralized control system for the processes with higher number of manipulated variables then the controlled variables. The method does not require knowledge of the dynamical mathematical model of the controlled system. All necessary information for the design can be obtained by simple evaluation of the steady-state experimental data. It is shown how to estimate the process gain matrix and how to calculate the matrices of the static compensator. 
The use of the static compensator does not eliminate the dynamical coupling of the individual control loops, nor does it ensure control stability. The main benefit is a general solution for the pairing of the manipulated and controlled variables for individual control loops in the case of multivariable systems. In addition, the use of the static compensator provides unit gain of each pair, the invariance in steady state and suppression of the offsets. These features make tuning of individual PID controllers easier.

\section{Acknowledgment}

This research was supported by SGS grant at Faculty of Electrical Engineering and Informatics, University of Pardubice.

\section{References}

1. Nordfeldt, P., Hägglung T.: Decoupler and PID controller design of TITO systems. Journal of Process Control, 10, p. 923-936 (2006).

2. Waller, M., Waller, J.B., Waller, K.V.: Decoupling Revisited. Ind. Eng. Chem. Res., 42, p. 4575-4577 (2003).

3. Dušek, F., Honc, D., Merta, J.: Static Compensator for Decentralized Control of Nonsquare Systems, Advances in Intelligent Systems and Computing, 1047, p. 1-6 (2019).

4. Skogestad, S., Postlethwaite, I.: Multivariable feedback control: analysis and de-sign. 2nd repr. ed. Chichester: Wiley, xiv, 574 s. (2008).

5. Lee, J., Kim, D.H., Edgar, T.F.: Static Decouplers for Control of Multivariable Processes. AIChE Journal, Vol. 51, No. 10, p. 2712-2720 (2005).

6. Skogestad, S., Morari, M.: Implications of large RGA elements on control elements. Ind. Eng. Chem. Fundam., 26, p. 2323-2330 (1987).

7. Bequette, B.W.: Process control: modeling, design, and simulation (Chapter 12. Ratio, Selective, and Split-Range Control). Upper Saddle River, N.J.: Prentice Hall PTR, xxix, 769 p. (2003). 